\title{
ON ITERATED LIMITS OF MEASURABLE MAPPINGS
}

Elias Zakon

(received July 1, 1964)

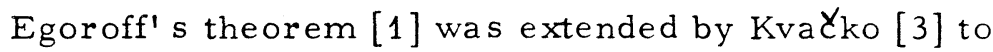
functions with values in a separable metric space; and, as is easily seen, this result applies also to separable pseudometric spaces. 1) In the present note we shall use this theorem to obtain some propositions on iterated limits, which, despite their simplicity, seem not yet to be known in the proposed generality.

Terminology and Notation. We shall denote by $m$ a non-negative countably additive measure defined on a $\sigma$-field $M$ of subsets of a set $S$. The triple $(S, M, m)$ is called a measure space, also briefly denoted by $S$. The measure $\mathrm{m}$ (and the space $S$ ) are called $\sigma$-finite if $S=\bigcup_{p=1}^{\infty} G_{p}$ for some sets $G_{p} \in M$, with $m G_{p}<\infty, p=1,2, \ldots$ A mapping (function) $f: S \rightarrow T$, where $T$ is a pseudometric space with pseudometric $\rho$, is said to be measurable if $f^{-1}(G) \in M$ for each open set $G \subseteq T$. By a double net we mean a family of functions $\left\{\mathrm{f}_{\mathrm{ij}}\right\}$ where the parameters $i$ and $\mathrm{j}$ run independently

1) The theorem in question is as follows: "If $\left\{\mathrm{f}_{n}\right\}$ is a sequence of measurable functions from a measure space $S$ $(\mathrm{mS}<\infty)$ into a separable pseudometric space $\mathrm{T}$, and if $f_{n} \rightarrow f$ almost everywhere on $S$, then $f_{n} \rightarrow f$ almost uniformly on $\mathrm{S}$ "(terminology and notation is explained below). Unfortunately, Kvačko's proof contains an error (Lemma 3, p. 89, is incorrect). This error can, however, be rectified, so that the theorem is still valid (we intend to show this in a separate paper [6] which gives also additional generalizations of the theorem).

Canad. Math. Bull. vol. 8, no. 1, February 1965 
over some directed sets ${ }^{2)} I$ and $J$, respectively. Such a net is said to be quasi-countable if each of the index sets $I$ and $J$ has a countable cofinal subset, with no last element. 3 ) A subsequence $\left\{\mathrm{f}_{\mathrm{i}_{\mathrm{n}}{ }_{\mathrm{n}}}\right\}(\mathrm{n}=1,2, \ldots)$ of a double net is cofinal iff, for any $i \in I$ and $j \in J$, there is $n$ such that $i_{n} \geq i$ and $j_{n} \geq j$. Convergence of nets and double nets is defined as usual (cf. Kelley, [2], p. 62 ff). A net, or a sequence, $\left\{f_{i}\right\}$, is said to converge almost uniformly on $S$ if, for every real $\varepsilon>0$, there is a set $D \subseteq S(D \in M)$ such that $m(S-D)<\varepsilon$ and $\left\{f_{j}\right\}$ converges uniformly or $D$; similarly for iterated limits (cf. Note 1 below). The term "almost everywhere on $S$ " or "at almost every $x \in S$ " means "everywhere, except for a set of measure $0^{\prime \prime}$. A space $T$ is separable iff it has a dense countable subset.

The theorems to be proved are as follows:

I. Let $f_{i j}: S \rightarrow T \quad(i \in I, j \in J)$ be a quasi-countable double net of measurable mappings from a measure space (S, M, m) into a separable pseudometric space $(T, \rho)$. Assume that $\mathrm{mS}<\propto$ and that the iterated limit

$$
\lim _{i} \lim _{j} f_{i j}(x)=g(x)
$$

exists at almost every $x \in S .4)$ Then there is a cofinal subsequence $\left\{f_{i n}\right\} \subseteq\left\{f_{i j}\right\}$ which converges to the function $g$

2) i. $\epsilon$, partially ordered sets in which any two elements have an upper bound.

3) A subset I' of a partially ordered set $I$ is cofinal iff, for every $i \in I$, there is $i^{\prime} \in I^{\prime}$, with $i^{\prime} \geq i$. An important example of a quasi-countable net $\left\{f_{i j}(\bar{x})\right\}$ is the case where $i$ and $j$ are parameters ranging continuously over all reals. The rationals form the countable cofinal subset.

4) For brevity, we use the "lim" notation also in pseudometric spaces (where the limit may not be unique). 
almost uniformly on $S$ as $n \rightarrow \infty$, and such that ${ }^{i}<i_{n+1}$ and $\mathrm{j}_{\mathrm{n}}<\mathrm{j}_{\mathrm{n}+1}, \mathrm{n}=1,2, \ldots$, under the order in $\mathrm{I}$ and $\mathrm{J}$, respectively.

II. Under the assumptions of Theorem I leven if $S$ is only $\sigma$-finite), there is a cofinal subsequence $\left\{\mathrm{f}_{\mathrm{i}_{n} \mathrm{j}_{n}}\right\}$ such that $i_{n}<i_{n+1}$ and $j_{n}<j_{n+1}, n=1,2, \ldots$, and such that

$$
\lim _{n \rightarrow \infty} f_{i} j_{n}(x)=g(x), \text { for almost every } x \in S
$$

III. If, in Theorems I and II, the index set I is of order type $\omega$ (i.e.,order-isomorphic to the set of positive integers), then the subsequence $\left\{f_{i j}\right\}$, postulated in these theorems, can be obtained in the stronger form $\left\{f_{i j}\right\}$, where $i$ ranges over the whole of $I$, and $j_{i}$ is a strictly increasing function of i. Moreover, in this case, the iterated limit (1) is almost uniform on $S$ (cf. Note 1 below), provided that $\mathrm{mS}<\infty$.

Proof of I. By quasi-countability, the index sets I and $J$ have countable cofinal subsets with no last elements (call them $I^{\prime}$ and ' $J^{\prime}$, respectively). As is easily seen, the subsets $I^{\prime}$ and $J^{\prime}$ can be chosen to be of order type $\omega .{ }^{5)}$ Since our problem consists only in selecting an appropriate subsequence from the net $\left\{f_{i j} \mid i \in I, j \in J\right\}$, we lose no generality by

5) Indeed, if, say, $I^{\prime}$ is countable but not of type $\omega$, we always can replace $I^{\prime}$ by a cofinal subset $I^{\prime \prime}=\left\{i_{n}^{\prime \prime}\right\}$

$(n=1,2, \ldots)$ of that type. For this purpose, write $I^{\prime}$ as a (not necessarily monotone) sequence $I^{\prime}=\left\{i_{n}^{\prime}\right\} \quad(n=1,2, \ldots)$ and then select from it an increasing subsequence $I^{\prime \prime}=\left\{\begin{array}{c}i^{\prime \prime} \\ n\end{array}\right\}$ such that $i_{n}^{\prime} \leq i_{n}^{\prime \prime}<i_{n+1}^{\prime \prime}(n=1,2, \ldots)$ under the ordering of I. Such a subsequence can be constructed inductively because $I^{\prime}$ is directed and has no largest element under its ordering inherited from I. 
replacing that double net by $\left\{f_{i j} \mid i \in I^{\prime}, j \in J^{\prime}\right\}$. Thus we may (and shall) assume that I and $J$ are themselves of type $\omega$; for simplicity, we identify each of them with the set of all positive integers in the usual order. Also, no generality is lost by assuming that the iterated limit (1) exists at each $x \in S$ (dropa set of measure zero!). Thus (1) becomes

$$
\lim _{i \rightarrow \infty} \lim _{j \rightarrow \infty} f_{i j}(x)=g(x) \quad \text { for each } x \in S
$$

with $i$ and $j$ taking only positive integral values. We are now in the situation described in Proposition III; thus we shall construct the required double subsequence right away in the stronger form $\left\{\mathrm{f}_{\mathrm{ij}}\right\}_{\mathrm{i}}$ as stated in (III).

$$
\text { For each fixed } \left.i, \text { let } \lim _{j \rightarrow \infty} f_{i j}(x)=g_{i}(x) .6\right) \text { Then (4) turns }
$$

into

$$
\lim _{i \rightarrow \infty} g_{i}(x)=g(x) \quad(x \in S)
$$

where the functions $g_{i}$ (and hence also $g$ ) are measurable, each being the pointwise limit of a sequence of measurable functions. 7)

Now, given any $\varepsilon>0$, formula (5), combined with the generalized theorem of Egoroff (see Footnote 1), yields a set $D_{0} \in M$ such that $m\left(S-D_{0}\right)<\frac{\varepsilon}{2}$ and $g_{i} \rightarrow g$ uniformly on $D_{0}$.

6) More precisely, we use the axiom of choice to select, for each $i$, one of the (possibly many) values of $\lim _{j \rightarrow \infty} f_{i j}(x)$, and call it $g_{i}(x)$.

7) The measurability of the limit function (well known for real functions) remains valid also for functions with values in a pseudometric space, as can easily be shown. 
Similarly, the formulae $f_{i j} \rightarrow g_{i}$ yield, for each $i$, a set $D_{i} \in M$ such that $m\left(S-D_{i}\right)<\varepsilon / 2^{i+1}$ and $f_{i j} \rightarrow g_{i}$ uniformly on $D_{i}$. Let $D=\bigcap_{i=0}^{\infty} D_{i}$. Then, clearly, $m(S-D)<\varepsilon$ and all the convergences $f_{i j} \rightarrow g_{i}$, as well as $g_{i} \rightarrow g$, are uniform on $D$. Letting here $\varepsilon=1 / k, k=1,2, \ldots$, we obtain, for each $k$, a set $D^{k} \in M$ such that $m\left(S-D^{k}\right)<1 / k$ and such that all the convergences $g_{i} \rightarrow g$ and $f_{i j} \rightarrow g_{i}$ are uniform on each $D^{k}$. Thus, given any positive integers $k$ and $n$, there is $i_{k n} \geq n$ such that $\rho\left(g_{i}, g\right)<\frac{1}{2 n}$ on $D^{k}$ for all $i \geq i_{k n}{ }^{8)}$ Moreover, by applying a double induction process, the $i_{k n}$ can be so selected that they increase with $k$ and $n$. Since $i_{n n} \geq i_{k n}$ when $n \geq k$, we have (writing, for simplicity, $i_{n}$ for $i_{n n}$ )

(8) $\rho\left(g_{i}, g\right)<\frac{1}{2 n}$ on $D^{k}$ whenever $i \geq i_{n}$ and $n \geq k$.

Similarly, from the uniform convergences $f_{i j} \rightarrow g_{i}$ on all $D^{k}$, we can find, for any positive integers $i, k, n$, an integer $j_{i k n}$ (increasing with $\left.i, k, n\right)$ such that $\rho\left(f_{i j}, g_{i}\right)<\frac{1}{2 n}$ on $D^{k}$ whenever $j \geq j_{i k n}$. Since $j_{i i i} \geq j_{i k n}$ when $i \geq n \geq k$, we certainly have (writing $j_{i}$ for $\left.j_{i i i}\right), \rho\left(f_{i j}, g_{i}\right)<\frac{1}{2 n}$ on $D^{k}$ whenever $j=j_{i}$ and $i \geq n \geq k$. Combining this with (8), we get (since $i_{n} \geq n$ )

$$
\rho\left(f_{i j}, g\right)<\frac{1}{n} \text { on } D^{k} \text { whenever } i \geq i_{n} \text { and } n \geq k
$$

8) For brevity, we write $\rho\left(g_{i}, g\right)$ for $\rho\left(g_{i}(x), g(x)\right)$. 
From this, however, it easily follows that the double subsequence $\left\{\mathrm{f}_{\mathrm{ij}}\right\}_{i}$ converges uniformly to $\mathrm{g}$ on each $\mathrm{D}^{\mathrm{k}}$. Since $m\left(S-D^{k}\right)<\frac{1}{k}(k=1,2, \ldots)$, the measure of the set $S-D^{k}$ can be made less than any prescribed $\varepsilon>0$. But this shows that $f_{i j_{i}} \rightarrow g$ almost uniformly on $S$, and the theorem is proved. Simultaneously, we have established (III) as far as it concerns (I)

NOTE 1. We have actually proved more than that. Indeed, formula (9) holds not only for $j=j_{i}$ but also for $j \geq j_{j}$. Thus we have shown that $\rho\left(f_{i j}, g\right)<1 / n$ on $D_{k}$ whenever $n \geq k, \quad i \geq i_{n}$ and $j \geq j_{i}$, where $i_{n}$ depends only on $n$, and $j_{i}$ depends only on $i$. It is natural to say that the iterated limit (1) is, in this case, uniform on each $\mathrm{D}_{k}$ and thus almost uniform on S. In this way the last part of (III) has, likewise, been established.

Proof of II. Suppose that $S$ is $\sigma$-finite, i.e., $S=\underset{p=1}{\bigcup} G_{p}$, with $\mathrm{mG}_{\mathrm{p}}<\infty\left(G_{\mathrm{p}} \in M\right), p=1,2, \ldots$ As in the proof of $I$, we may identify the index sets $I$ and $J$ with the set of all positive integers and apply Egoroff' $s$ theorem to each of the sets $G_{p}$ (each being of finite measure!). Introducing, as before, the functions $g_{i}(i=1,2, \ldots)$, we obtain, for each positive integer $p$, a sequence of sets $D_{p}^{q} \subseteq G_{p}\left(D_{p}^{q} \in M\right.$, $q=1,2, \ldots$, such that $m\left(G_{p}-D_{p}^{q}\right)<1 / q$ and such that the

9) Indeed, if the index set I is of type $\omega$, the proof given above requires only a selection of a cofinal $\omega$-type subset $\mathrm{J}$ ' from $\mathrm{J}$. Thus it yields the required subsequence in the stronger form $\left\{f_{i j}\right\}$. 
convergences $g_{i} \rightarrow g$ and $f_{i j} \rightarrow g_{i}$ are uniform on each of the sets $D_{p}^{q}(p, q=1,2, \ldots)$. Moreover, we have $m\left(G_{p}-\bigcup_{q=1}^{\infty} D_{p}^{q}\right)=0$ and hence, setting $D=\bigcup_{p=1}^{\infty} \bigcup_{q=1}^{\infty} D_{p}^{q}$, $m(S-D)=m\left(\bigcup_{p=1}^{\infty} G_{p}-\bigcup_{p=1}^{\infty} \underset{q=1}{\bigcup} D_{p}^{q}\right) \leq m \underset{p=1}{\bigcup}\left(G_{p}-\bigcup_{q=1}^{\infty} D_{p}^{q}\right)=0$. Thus, to complete the proof, it suffices to construct a subsequence $\left\{f_{i j_{i}}\right\} \quad(i=1,2, \ldots)$ such that $\lim _{i \rightarrow \infty} f_{i j}(x)=g(x)$ at each $x \in D$. For this purpose, we renumber the sets $D_{p}^{q}$ (which form a countable set family), putting them into a single sequence $\left\{D^{k}\right\} \quad(k=1,2, \ldots)$ comprising all $D_{p}^{q}(p, q=1,2, \ldots)$. Then, proceeding exactly as in the proof of (I), we obtain a subsequence $f_{i j}$ which converges uniformly to $g$ on each $D^{k}$ and, hence, converges pointwise at each $x \in D=\bigcup_{k=1}^{\infty} D^{k}$. This completes the proof.

NOTE 2. Simultaneously, we have established the remaining part of (III).

Final Remarks. As has already been mentioned, any family of functions $f(i, j, x)$, where $i$ and $j$ are continuous real parameters, is a special case of a quasi-countable net, in which the index sets $I$ and $J$ coincide with the set of all real numbers in their usual order, while the rationals can serve as the required countable cofinal subset. Thus our theorems cover the case of an iterated $\operatorname{limit} \lim \lim f(i, j, x)$

$$
i \rightarrow \infty \quad j \rightarrow \infty
$$

where $i$ and $j$ tend to $+\infty$, in the sense of the standard topology of the real number system. The case where one or both of $i$ and $j$ tend to $-\infty$ is covered by simply reversing the order in $I$ or $J$ or both. Also covered is the case of one-sided limits $i \rightarrow p-, i \rightarrow p+$, etc., with $p$ a finite number; we then let I (or $\mathrm{J}$, or both, as the case may be) be the set 
of all reals less than $\mathrm{p}$ (respectively, greater than $\mathrm{p}$, with the order reversed, etc.). By combining two one-sided limits $(i \rightarrow p-$ and $i \rightarrow p+)$ one can obtain bilateral limits $(i \rightarrow p)$; we then let $I$ consist of all pairs of reals $(x, y)$, with $x<p$ and $y>p$, ordered as follows: $(x, y) \leq\left(x^{\prime}, y^{\prime}\right)$ iff $x \leq x^{\prime}$ and $y^{\prime} \leq y$. Thus all possible combinations of finite and infinite, one-sided and two-sided, limits on the real axis are included as special cases. 10) Note that, if the parameter $i$ ranges only over positive integers, then Theorem III applies. This means that the iterated limit (1) can be transformed into a simple limit by making $j$ an increasing function of $i$.

The problem of transforming an iterated limit into a single one is not new. For double nets in a topological space, a well known variant of the diagonal process yields a subnet which converges to the given iterated limit (cf. Kelley, [2], p. 69). 11) As compared with this process, our theorems yield a stronger result, namely a cofinal subsequence with the required property. ${ }^{12}$ ) [It is true, this result was made possible

10) This is noteworthy inasmuch as the theorem of Egoroff itself cannot be extended to families of functions depending on a continuous real parameter, as was shown by Tolstov [4] and Weston [5].

11) The net SoR in Kelley's Theorem 4 (p. 69) is, in general, not a subnet. It becomes, however, a subnet if all directed

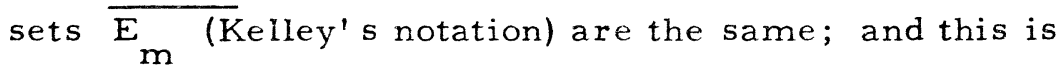
exactly the case of a double net as considered in the present note (in our notation, $E_{m}=J$ ).

12) We must qualify Kelley's assertion (p. 69) that "considering double sequences, no sequence whose range is a subset of $\omega \times \omega$ can have that property". Our proof shows that such a sequence may well exist, under appropriate assumptions. 
only by the additional assumptions of quasi-countability and $\sigma$-finiteness.] Moreover, our theorems deal with double nets of mappings, not just elements of a topological space. This leads to assertions as to the uniformity or "almost uniformity" of the convergence, as stated in our Theorems I and III. For these purposes, the process of Kelley's Theorem 4 (p. 69) does not suffice.

\section{REFERENCES}

1. D. T. Egoroff, Sur les suites de fonctions mesurables, C. R. Acad. Sci. Paris, 152 (1911), 244-6.

2. J. Kelley, General Topology, D. Van Nostrand, N. Y., 1960.

3. M. E. Kvačko, On measurable mappings of spaces (Russian, English summary), Vestnik Leningr. Univ. 13 (1958), no. 13, 87-101. (Quoted in Math. Reviews, 1959, no. 5267, p. 873).

4. G. Tolstov, Une remarque sur le théorème de $D$. Th. Egoroff, Doklady Acad. Nauk SSSR (N.S.) 60 (1948), 973-5.

5. J.D. Weston, A counterexample concerning Egoroff's theorem, J. London Math. Soc. 34 (1959), 139-140.

6. E. Zakon, On "essentially metrizable" spaces and on measurable functions with values in such spaces, (to appear in the Transactions, Am. Math. Society).

University of Windsor,

Summer Research Institute of the Canadian Math. Congress 